\title{
La evaluación por competencias
}

\section{José Carlos Escobar Hernández* \\ CEPE-Taxco}

\begin{abstract}
Resumen: Este artículo se ocupa del tema de la evaluación por competencias. Si bien aborda asuntos generales del correspondiente enfoque -de enorme interés en los cursos de formación de profesores en el CEPE- presenta sobretodo en diversos aspectos de la evaluación. Se habla de las capacidades, que en conjunto dan por resultado una determinada competencia y se adopta una postura utilitaria en la definición de competencia y algunos de los presupuestos básicos que la definen. Se comentan además los conceptos de conocimiento y adquisición, la diferencia entre saber y conocer, capacidad innata vs. capacidad adquirida, inteligencias múltiples y talento, todo lo cual hace competentes a las personas. Se ofrecen algunas definiciones de competencia y los elementos que las componen para comentar sobre su aplicación en la solución de problemas en el área de la formación de profesores de español como lengua extranjera. Se abordan las características principales de la evaluación por competencias y se presentan ejemplos, para concluir con la mención de los criterios que sirven de fundamento a las competencias, los elementos que requiere la evaluación e instrumentos para aplicarla.
\end{abstract}

Palabras clave: competencia, evaluación, capacidad, conocimiento, problema.

\begin{abstract}
This article deals with the issue of competency assessment. Although it addresses general issues related to the approach itself which is at the center of the academic interest of the teacher training courses of CEPE-, it basically presents different aspects related to evaluation. It deals with abilities, which all in all result in certain competencies, from a practical stance in order to define competency and to describe some of its basic and defining assumptions. Concepts like knowledge and acquisition, the difference between knowing and being familiar with, innate vs. acquired skills, multiple intelligences and talent, which make a person competent, they are all dealt with in this article. Some definitions of competency are offered together with
\end{abstract}

*Profesor de ELE desde 1986 (CEPE-UNAM). Doctor en Educación, Maestro en Filosofía Antropológica y Licenciado en Letras inglesas. Co-autor de libros de texto del CEPE en CDMX y San Antonio, Texas. Profesor de formación de profesores de español. Líneas de investigación: interculturalidad, lexicología y traducción. 
the elements they are made of in order to talk about their application in solving real-life problems in the specific field of Spanish teacher training. The characteristics of competency assessment are also dealt with here, together with examples. It concludes with a mention of the criteria which are the very basis of a competency, the elements required in its evaluation, and some means to implement it.

Keywords: competency, evaluation, skills, knowledge, problem.

INTRODUCCIÓN

Mucho se habla del desarrollo de competencias y las hay de todo tipo (procedimentales, afectivas, socioculturales, lingüísticas, interculturales, escolares, etc.), pero es posible que no se las considere adecuadamente al elaborar un currículo o programa de estudios en el cual pueden dejarse fuera diversas capacidades que, en conjunto, han de dar como resultado una(s) determinada(s) competencia(s). Quizá fue esto lo que llevó al Dr. Ángel Díaz Barriga a escribir el artículo denominado "El enfoque por competencias en la educación: ¿Una alternativa o un disfraz de cambio? “"(2006), en el cual señala que las aplicaciones del enfoque por competencias en educación suelen ser parciales y la generación de orientaciones "más o menos apresuradas son insuficientes para promover el cambio educativo que se busca y su impacto prácticamente inexistente en las prácticas educativas" (p. 8).

No obstante, dado que constantemente se alude a las competencias para la vida que la ONU ha propuesto para garantizar que un individuo sea capaz de aplicar sus conocimientos, destrezas y sentimientos en la solución de un problema, así como el enorme número de publicaciones sobre las competencias y su aplicación en el diseño de cursos y programas, conviene revisar algunos de sus presupuestos básicos para pasar después al tema de la evaluación basada en competencias, parte esencial de un currículo que -como sucede en el caso del Diplomado de Especialización en la Enseñanza del Español como Lengua Extranjera (EEELE) que imparte el CEPE-UNAM- busca formar maestros competentes, según se indica en la Presentación del mismo:

La especialización tiene como objetivo formar profesores de español como lengua extranjera con amplios conocimientos teórico-metodológicos que sean capaces de ofrecer una respuesta innovadora, ética e independiente a las demandas educativas en su campo (compruébese en la siguiente dirección: http://especializacion.cepe.unam.mx/web/presentacion.php ). 
La frase "que sean capaces" alude al concepto de las competencias, lo cual se confirma al ver que el correspondiente Plan de estudios contiene saberes que llevan tanto a la aplicación como a la práctica de los conocimientos que conforman el Diplomado, todo lo cual constituye la declaración final de lo que debe producirse mediante dicho plan de estudios. Pero, si bien las competencias de formación profesional suelen ser enunciadas en objetivos comportamentales y como "habilidades y destrezas para la vida", conforme la definición que da la OCDE para el examen PISA (2005), no hay que olvidar que las competencias se desarrollan a lo largo de la vida -como bien anota Días Barriga (o.c., p. 15) - y que en realidad se trata de procesos prolongados, porque su desarrollo puede mejorarse permanentemente, lo cual debe tomarse en cuenta al evaluarlas.

De acuerdo con Núñez Chan (2003, p. 1-2), el proceso al diseñar el currículo desde la perspectiva de las competencias exige reconocer problemáticas susceptibles de intervención, la identificación de núcleos problemáticos, perfiles profesionales y competencias clave, así como el reconocimiento de las funciones integradoras, los ámbitos para su desempeño, y el diseño de un mapa curricular que considere trayectorias en el desarrollo de tales competencias. Un campo profesional se define por sus problemas específicos, las competencias que exige, sus funciones y ámbitos de acción, una visión disciplinar, lo mismo que por las prácticas presentes y potenciales a las que se enfrenta el profesionista.

\section{AlgunAs PRECISIONES}

Según Delors (1994, p. 91-103), el complejo mundo en que vivimos exige que la educación no se limite a la mera acumulación de conocimientos sino a la acción, para que el individuo pueda estar en condiciones de aprovechar y utilizar sus saberes, actualizándolos, profundizándolos y enriqueciéndolos para adaptarse a un mundo en permanente cambio. Por tal razón, señala que la educación debe estructurarse alrededor de cuatro aprendizajes (saberes) fundamentales:

1) aprender a conocer, es decir, adquirir los instrumentos de comprensión. Este saber se relaciona con el conocimiento, los contenidos conceptuales o declarativos,

2) aprender a hacer para influir sobre el entorno. Este saber se relaciona con los contenidos procedimentales o de habilidades, los cuales se orientan a la ejecución de una acción o procedimiento, a las destrezas necesarias para la realización de una actividad. 
3) aprender a vivir juntos para participar y cooperar con los demás. Este saber se relaciona con los contenidos actitudinales o valorativos, a las actitudes que apuntan al desarrollo de la formación personal y social para un desempeño adecuado en un contexto determinado.

Cabe señalar que el cuarto de los saberes que propone dicho autor (aprender a ser) es un proceso que recoge los tres aprendizajes anteriores y constituye propiamente una competencia originaria, es decir, que inicia y da lugar a las demás. Sin embargo, los aprendizajes requieren también de ciertas aptitudes, por lo cual es necesario distinguir entre conocimiento y capacidad para contar con una noción clara de lo que es una competencia.

¿QUÉ SE ENTIENDE POR CONOCIMIENTO?

Es el acopio de información (datos) y suele entenderse entre otras cosas como: (a) hechos o información adquiridos a través de la experiencia y la relación con el entorno o de la educación escolar, (b) lo que se adquiere como contenido intelectual relativo a un campo determinado por intermedio de la razón, el manejo de conceptos, todo lo cual se almacena en la memoria. Su adquisición implica procesos cognitivos que implican aspectos como la motivación, las emociones, la percepción, las sensaciones, la conceptualización, la adquisición de palabras y el manejo del lenguaje, la socialización, la asociación (inducción), el razonamiento (deducción), etc. De tal suerte, hay muchos tipos de conocimiento: como la mera acumulación de experiencia, la lengua, las leyendas, las costumbres, ideas y creencias de una cultura particular, la propia historia, el arte, la artesanía, la ciencia, el conocimiento escolar, etc.

Según Quezada (1998, Cap. 3) diferenciamos de un modo técnico y formalizado los conceptos de conocer y saber: el primero se refiere a una evidencia basada en la experiencia y la memoria, en tanto que el segundo requiere además estar en relación con un sistema coherente de significado y de sentido, fundado en lo real y comprendido como realidad. El uso del lenguaje ilustra el sentido de ambos fenómenos cognitivos: conocemos los lugares y a las personas, y así decimos "conozco a Mario" y "conozco Taxco“. En cambio decimos "sé montar en bicicleta", "sé hablar japonés", "sé tocar el piano". En algunos casos la diferencia tiene sentido pues no es lo mismo decir "conozco los autos alemanes" o "conozco el teorema de Pitágoras" versus "sé cómo funcionan los autos alemanes" o "sé el teorema de Pitágoras". Una cosa es haber oído hablar de algo y otra saberla o comprenderla en todo su sentido o contenido. No es 
lo mismo tener referencias vagas y superficiales sobre algo que saber a fondo sobre algo. Si bien no es una regla, podría decirse que conocer refiere a una experiencia surgida del contacto físico y material, en tanto que saber refiere a una experiencia debida a los mecanismos cognitivos propios de la inteligencia. De ahí el sentido de "saber ser", "saber hacer", "saber conocer" y "saber convivir", saberes que requieren de capacidad para realizarlos. Además, el saber se alimenta de visiones disciplinarias que por supuesto avanzan y modelan los contenidos, las habilidades, y los paradigmas desde los cuales pueden ser vistos y planteados a manera de problemas (Núñez Chan, o.c., p. 2).

\section{¿QUÉ SE ENTIENDE POR CAPACIDAD?}

Es la predisposición para resolver problemas o llevar a cabo una determinada tarea o cometido y es un factor que vincula una habilidad con un conocimiento. Es un saber delimitado, más pequeño que una competencia, que se desarrolla y se pone en acción para desarrollar una competencia. Las capacidades hacen sinergia entre ellas y pueden ser cognitivas, relacionales, afectivas, actitudinales, de conocimientos, de procedimientos, etc. Es el conjunto de recursos que tiene un individuo para desempeñar una determinada tarea.

Hay capacidades innatas, de las cuales se puede estar o no consciente y que ayudan a enfrentar las tareas que surgen en la vida, y capacidades adquiridas o aprendidas, las cuales una vez dominadas se realizan sin pensar en ellas y se le conoce como destreza o habilidad. Así, si no se cuenta con una capacidad pulmonar suficiente, no será fácil tocar instrumentos de viento, o si por otro lado se carece de elasticidad, será difícil ejecutar las elegantes formas que requiere una actividad como el ballet. Las capacidades innatas son quizá las más importantes, en la medida en que posibilitan las demás. Esto se relaciona directamente con las inteligencias múltiples que poseen los individuos (Gardner: 1983) por ejemplo, el aprendizaje de una ciencia requiere de un mínimo de inteligencia lógico-matemática y lingüística, la convivencia armónica con los demás requiere de un mínimo de inteligencia interpersonal, la propuesta de un cambio o mejora requiere de un mínimo de inteligencia creativa, etc.

Por otro lado, el concepto de capacidad tiene una estrecha relación con los de talento e inteligencia porque cuando el individuo sobresale y manifiesta talento en un tema, en una materia o en una actividad, y la completa con éxito, se le considera capaz e idóneo para llevarla a cabo. Así, cuando decimos que "José es capaz de resolver conflictos de una manera pacífica" o que "Ana es capaz de enseñar el español a los extranjeros", estamos hablando de su capacidad para resolver conflictos (en el caso de José) y de enseñar el español (en el caso de 
Ana). En cualquiera de los casos, no se está diciendo que José y Ana sólo tienen conocimientos o que sólo tienen habilidades cognitivas pues:

a) en el caso de José, tiene que tener además aptitudes o habilidades socioemocionales, como la empatía, precisamente para solucionar los problemas de una manera pacífica.

b) Y, en el caso de Ana, debe de contar con aptitudes o habilidades sociales e intrapersonales, como la competencia intercultural, así como una fuerte imaginación para pensar en situaciones que puede presentar a sus alumnos para aclarar o ejemplificar el significado, el uso y el sentido de lo expresado en español.

De tal suerte, los conocimientos adquiridos y las capacidades innatas y adquiridas por ambos los hacen competentes.

¿QUÉ SE ENTIENDE POR COMPETENCIA?

Es la eficacia para resolver problemas (del latín competentia-"corresponder a", "hacer bien", "ser bueno en algo", de ahí "ser competente"). Una competencia supone un actuar, pero un actuar complejo, para actuar sobre la realidad; es decir, con un propósito o para resolver un problema. Sobre el término se han dado definiciones muy diversas y entre las muchas que hay se encuentran, por ejemplo, las de:

- Mertens (2000), citado por Sergio Tobón (2015) en su obra "Formación Basada en Competencias-Pensamiento complejo, diseño curricular y didáctica", afirma que la definición de una competencia se centra en los comportamientos constatables de una persona que facilitan el desempeño eficiente de sus tareas laborales. No se trata sólo de lo que hacen, sino de cómo lo hacen.

- Perrenoud (2004) las define como una "Síntesis combinatorias de procesos cognitivos, saberes, habilidades, conductas en la acción y actitudes, mediante las cuales se logra la solución innovadora a los diversos problemas que plantea la vida humana y las organizaciones productivas".

- Zabala (2005, p. 40) plantea que la competencia es la "capacidad de enfrentarse con garantías de éxito a tareas simples y complejas en un contexto determinado".

- Prosser (2014) la define como un "Sistema de conocimientos, habilidades, valores, criterios, aptitudes personales y sociales que son re- 
queridas para el desempeño exitoso de una profesión, función o cargo y para el logro de resultados y metas en contextos determinados".

Para Díaz Barriga (o.c., p. 20) en una competencia se combinan tres elementos: (a) una información; (b) el desarrollo de una habilidad y (c) su puesta en acción en una situación inédita, pues se requiere del dominio de una información específica, el desarrollo de una habilidad o una serie de habilidades derivadas de los procesos de información en una situación problema, donde la competencia se puede generar.

Se puede pensar en una competencia como un comportamiento clave para la competitividad (perspectiva conductista) o como los atributos que permiten cumplir con los requerimientos laborales y profesionales (planteamiento funcionalista), pues el enfoque enfatiza que no tiene sentido ningún contenido escolar si éste no se aprende en un contexto real. Por ello Perrenoud, uno de los promotores actuales del enfoque por competencias, habla de la necesidad de "movilizar la información" en el proceso de la educación o aprendizaje (1999, p. 61) y señala que este enfoque permite materializar la perspectiva de Piaget sobre la conformación de los esquemas de acción y, al mismo tiempo, se acerca al enfoque basado en la resolución de problemas, un planteamiento que se opone al enciclopedismo en la educación.

En el terreno específico de la formación de profesores de español como lengua extranjera se puede pensar sin mayores complicaciones en una definición de competencia entendida como "la capacidad de actuar de manera eficaz en una situación particular, donde dicha capacidad se apoya en los conocimientos adquiridos de un individuo para dar solución a un problema“ (Perrenoud: 2008). También se puede advertir que los conocimientos y recursos cognitivos se movilizan para poder dar solución a determinadas situaciones (Roegiers: 2007) --de enseñanza, en el caso de la enseñanza de lenguas--. Por ende, es necesario que el docente de un idioma desarrolle situaciones didácticas significativas, interactivas y orientadas hacia los objetivos de aprendizaje reales que promuevan el aprendizaje en sus alumnos.

Si toda competencia supone la movilización de saberes de diferente tipo -la movilización de conocimientos, el desarrollo de habilidades cognitivas, socioemocionales, determinadas disposiciones afectivas, actitudes, técnicas y procedimientos-, entonces es necesario que los ejercicios o actividades de un programa de enseñanza o formación no sean rutinarios ni simulados, a fin de llevar a los educandos a la formación (o desarrollo) de las habilidades o destrezas que se pretende desarrollar en ellos. De ahí la observación de Perrenoud en el sentido de que: 


\begin{abstract}
"Un médico, un arquitecto, un ingeniero, utilizan grandes fragmentos de numerosas disciplinas escolares y universitarias, pero también saberes constituidos, propios de su dominio de acción, ya sea saberes eruditos, profesionales o basados en la experiencia" (1999, p. 53).
\end{abstract}

\title{
CONSIDERACIONES ADICIONALES
}

No debe perderse de vista que el término competencias tiene un sentido utilitario en el mundo del trabajo, donde se le concibe como una estrategia que se apoya fundamentalmente en el análisis de tareas específicas, a partir de la cual se busca determinar las etapas puntuales en las que se debe formar a un individuo en la adquisición de las habilidades y destrezas que le permitan un desempeño eficiente en su labor.

Lo anterior no constituye un aspecto desdeñable en el terreno de la educación porque al final de cuentas -como se explicó en la Introducción- lo que se busca en la impartición de los Diplomados del CEPE, por ejemplo, es formar profesores capaces de enseñar el idioma español a los extranjeros. De tal suerte, siendo congruentes con el desarrollo de un programa basado en actividades o tareas que en su conjunto dan a los futuros profesores las competencias adecuadas para realizar esta labor, es necesario aplicar una evaluación acorde con el enfoque asumido. Por ende, es importante señalar las características de la evaluación por competencias, conforme a las siguientes preguntas y elementos.

¿CUÁL ES EL ESPÍRITU DEL ENFOQUE POR COMPETENCIAS?

Para aprender a andar en bicicleta, hay que subirse a la bicicleta y pedalear. Plantear la enseñanza con base en el desarrollo de competencias implica enseñar diversos conocimientos y técnicas, además de desarrollar una actitud y una habilidad para su aplicación (redactar una carta o ensayo, llevarse bien con los demás, preparar una clase, planear una tarea, atender a un paciente, etc).

¿PARA Diseñar un PROGRAMA POR COMPETENCIAS, LO PRIMERO ES DEFINIRLAS?

No, lo primero es definir los problemas: primero debe analizarse el problema y de ahí definir -como se ha hecho en el caso de los Diplomados de formación de profesores en el CEPE- cuáles son las competencias lingüísticas, socioculturales, gramaticales e interculturales que necesitan los maestros en ciernes y que se requieren para el adecuado desempeño de su futura labor profesional, 
etc. De tal suerte, el programa queda conformado por las diversas capacidades que llevarán a una(s) cierta(s) competencia(s).

¿QuÉ SE ENTIENDE POR “PROBLEMA“?

De acuerdo con la RAE, un problema es: (a) una cuestión que se trata de aclarar, (b) una dificultad de solución dudosa, o bien (c) un conjunto de hechos o circunstancias que dificultan la consecución de algún fin. Es una cuestión discutible, que necesita ser resuelta, de manera que existen muy distintos tipos de problemas: una tubería descompuesta, un cálculo diferencial, la productividad de una empresa, la calidad de vida o una guerra, comunicarse en otro idioma, etc. Asimismo, hay problemas personales, religiosos, políticos, científicos, sociales, económicos, financieros, etc., pues un problema puede presentarse en cualquier área de la existencia, la acción y el conocimiento humano.

No obstante, en el terreno de la enseñanza en general o de la enseñanza de lenguas en particular, y considerado desde el enfoque por competencias, un problema es cualquier tema referido a una habilidad o destreza, entendidos como la aplicación efectiva de los contenidos contemplados en un curso, programa o plan de estudios, como se puede ver en los dos ejemplos que se presentan a continuación.

\section{PROBLEMA 1: EL PROCESO DE LA REDACCIÓN}

Este problema es común a todos los planes y programas de enseñanza. Se trata de asegurar que los alumnos tengan la capacidad de redactar e incluso de hacerlo de una manera creativa, lo que además, cuando se trata del aprendizaje de una lengua extranjera, resulta sumamente difícil para los estudiantes por el manejo de vocabulario y elaboración sintáctica de las expresiones que requiere esta actividad.

El proceso consta de tres fases principales: (a) una planificación (inicio, mitad, final), (b) una textualización (poner en papel las ideas, citar adecuadamente, etc.) y (c) una revisión (puntuación, conectores adecuados, etc.).

Para resolver este problema ("el proceso de la redacción") es necesario contar con habilidades cognitivas y disposiciones afectivas porque si no tenemos disposición para revisar lo escrito, entonces faltará esa capacidad y por tanto 
habrá una incompetencia, dado que se estaría dejando incompleto el proceso que conlleva llegar a un producto final (el texto).

\title{
PROBLEMA 2: COMPRENSIÓN DE LECTURA
}

\begin{abstract}
Este problema es de interés fundamental en la enseñanza de lenguas e igualmente en la educación en general dado que la alfabetización, aprender a leer y comprender lo leído, es una tarea fundamental en la vida, en el desarrollo personal y profesional, además de que la capacidad de comprender una lectura debe abarcar textos escritos de diverso tipo y complejidad.
\end{abstract}

De ahí que todo maestro (de lengua) se pregunte cómo llevar al estudiante a esa competencia, pregunta que tiene como respuesta dotarlo de las capacidades necesarias para hacerlo, a saber: el manejo de vocabulario, el interés y familiaridad con en el tema, la comprensión de las ideas principales y secundarias, la capacidad para entender el interés y postura del autor, la habilidad para relacionar los hechos contenidos en al lectura con los conocimientos previos, el uso de ciertas palabras en el contexto presentado por la lectura, y en general la capacidad de reflexión para derivar de ello una opinión propia, etc.

¿QUÉ SE NECESITA PARA DESARROLLAR UNA COMPETENCIA?

Para lograrlo es necesario:

- Partir de situaciones desafiantes (cotidianas, reales o creadas ex profeso).

- Ir de lo general a lo particular y viceversa (para aterrizar la competencia a la realidad del alumno).

- Construir significativamente el conocimiento (debe ser útil, tener un sentido).

- Buscar que los conocimientos sean transferibles y aplicables.

- Desarrollarlas a largo plazo y progresivamente.

- Los conocimientos deben relacionarse con situaciones problemáticas (casos específicos que luego serán transferibles a otras realidades o problemas). 
¿QUÉ CRITERIOS SIRVEN DE FUNDAMENTO A LAS COMPETENCIAS?

Debe tomarse en cuenta que las competencias:

- Se componen de conocimientos explícitos y tácitos, en contextos concretos de actuación.

- Se encuentran en permanente desarrollo y su evaluación debe ser continua dado que toda competencia debe experimentar una evolución y mejora.

- Su desarrollo debe entenderse como un proceso de adaptación creativa en cada contexto determinado, en situaciones o problemas específicos.

- Varían en su desarrollo y nivel de logro según los grados de complejidad y de dominio por parte del individuo.

- Se desarrollan mediante procesos de significación para que un saber susceptible de enseñarse se transforme en un saber enseñado y, por lo tanto, esté disponible para que sea movilizado en cuanto se lo requiera.

\section{ELEMENTOS PARA LA EVALUACIÓN POR COMPETENCIAS}

Básicamente, los elementos que necesitan ser considerados para determinar un problema y después llevar a cabo una evaluación por competencias son:

1. La determinación del problema

2. Un verbo de desempeño

3. El objeto conceptual

4. La finalidad de la competencia

5. El(los) criterio(s) de calidad

6. Una condición de referencia

7. Las actividades-entrenamiento (evidencias-informes)

La evaluación surge y se concentra en la consideración puntual del punto No. 7 que se refiere a todas las actividades que darán lugar al entrenamiento, capacitación y formación de los aprendientes, junto con sus correspondientes evidencias e informes. Dicho de otra manera, el programa debe describir los conocimientos y destrezas que han de llevar a las competencias buscadas. 
Los dos problemas siguientes fueron redactados prácticamente con las mismas palabras para demostrar la manera en que se puede redactar de una manera básica un problema, independientemente del área o campo del saber en el que se trabaje. Por supuesto, no importa el orden de los elementos consignados, lo fundamental es que todos sean considerados.

EJEMPLO 1 - APLICADO AL ÁREA ODONTOLÓGICA

Problema: "Queremos dotar a los futuros dentistas de las herramientas adecuadas para que atiendan de la mejor manera las enfermedades dentales de la población mexicana“. (UNAM)

Verificación de los elementos del Problema.- Como se puede ver, la enunciación del problema contempla todos los elementos señalados como indispensables: (a) un verbo de desempeño ("Queremos dotar a los futuros dentistas"), (b) el objeto conceptual ("de las herramientas adecuadas"), (c) la finalidad de la competencia ("para que atiendan"), (d) el criterio de calidad ("de la mejor manera") y (e) una condición de referencia ("las enfermedades dentales de la población mexicana"). Ejemplos de actividad: el llenado de la historia clínica, la comunicación afectiva con el paciente, la orientación al paciente, y muchas otras más.

EJEMPLO 2 - APLICADO A LA FORMACIÓN DE PROFESORES DE ESPAÑOL COMO LENGUA EXTRANJERA

Problema: "Queremos dotar a los futuros profesores de español de las herramientas adecuadas para que apliquen de la mejor manera el enfoque comunicativo de enseñanza de lenguas".

Verificación de los elementos del problema.- Al igual que en el ejemplo 1 , se observa que el problema contiene todos los elementos requeridos al contener: (a) un verbo de desempeño ("Queremos dotar a los futuros profesores de español"), (b) el objeto conceptual ("de las herramientas adecuadas"), (c) la finalidad de la competencia ("para que atiendan"), (d) el criterio de calidad ("de la mejor manera") y (e) una condición de referencia ("el enfoque comunicativo de enseñanza de lenguas").

Ejemplos de actividad: la presentación de un tema, la aplicación de un examen diagnóstico, el diseño de un ejercicio, la preparación de temas de conversación, las dinámicas en el aula, la reconstrucción de un texto, etc. 
INSTRUMENTOS DE EVALUACIÓN

Evaluar por competencias supone el diseño de instrumentos en los que el estudiante demuestre con ejecuciones (evidencias) que puede realizar las tareas de la competencia exigida. Una reflexión, análisis o actividad integradora de carácter meramente teórico -referidos a la parte conceptual del conocimiento brindado por un programa- es contraria a los objetivos y finalidad que persigue el enfoque por competencias. La Universidad de Barcelona, por ejemplo, considera indispensable considerar y definir las herramientas de evaluación que deberán emplearse para este fin dado que son éstas las que proporcionan al estudiante recursos para la aplicación práctica de los contenidos aprendidos y facilitan la comprensión de los procedimientos y conceptos comprendidos en la materia (consúltese la bibliografía).

Como este no es el espacio para hablar del tipo de actividades que requiere el enfoque -el mapeo de las capacidades que progresivamente llevarán al desarrollo de una competencia determinada-, sólo cabe reiterar que cada curso del programa debe contemplar actividades para ejercitar y aplicar de manera práctica los contenidos, además de los recursos necesarios para llevarlas a cabo, lo cual es parte es parte del desempeño de los tutores a cargo de los cursos.

Por consiguiente, hay que considerar algunas de las herramientas que se pueden utilizar, a saber:

- Los cuestionarios se pueden usar para una evaluación formativa, sumativa y final, para comprobar el nivel de comprensión del contenido de las diferentes lecciones del curso y de las acciones exigidas por determinados problemas.

- La evaluación por pares consiste en hacer que los aprendientes revisen y comenten sus trabajos entre sí, que cada participante observe y valore el trabajo de al menos otros dos o tres compañeros. Es muy importante anotar que el tutor debe proporcionar instrucciones claras de lo que tienen que valorar y unas buenas rúbricas, las cuales deben ser sencillas porque aún no son expertos en el tema.

- De la misma manera, se puede abrir un foro de evaluación por pares para que los estudiantes puedan aportar sus comentarios, contribuyendo así a que el aprendizaje colaborativo y la retroalimentación sean enriquecedores.

- El debate permite la comunicación entre aprendientes y tutores, además de que constituye un buen apoyo para observar los niveles de enseñanza y de aprendizaje logrados.

- La demostración -como elemento esencial- permite evidenciar 
la(s) competencia(s) adquiridas dado que permiten mostrar la técnica utilizada en el ejercicio de una competencia y se verifican mediante la observación. Las evidencias son pruebas reales, observables y tangibles de las consecuencias del desempeño mostrado.

Niveles de EVALUACIÓN Y EJEMPLO finAL

En el enfoque por competencias, la evaluación se lleva a cabo por niveles y bajo criterios de desempeño, entendidos como el estándar o calidad de ejecución de una competencia que es uniforme para todos. Por tanto, el desarrollo de tales criterios debe ser puntual, crítico, objetivo y bien establecido para una determinada competencia, a fin de asegurar la confiabilidad y validez de su evaluación. De tal suerte, son cuatro los niveles de evaluación que se consideran:

- Nivel receptivo (sabe la teoría pero no la sabe aplicar)

- Nivel básico (resuelve bajo supervisión, con ayuda)

- Autónomo (resuelve solo pero únicamente en ciertos casos)

- Estratégico (resuelve con todos los criterios, en todos los contextos y con el protocolo necesario)

EJEMPLO FINAL APLICADO AL CONTENIDO DE UN CURSO DE ELE: LA COMPETENCIA LÉXICA

Problema: Queremos enseñar a los alumnos a manejar adjetivos y sustantivos para construir expresiones correctas en su comunicación en español.

Verificación de los elementos: "Queremos enseñar a los alumnos" (problema); a manejar (verbo de desempeño); adjetivos y sustantivos (objeto conceptual); para construir expresiones (finalidad de la competencia); correctas (criterio de calidad): en su comunicación oral y escrita (condición de referencia).

Ejemplo de actividad (colocaciones): En esta actividad se presenta a los alumnos un cuadro de colocaciones a base de adjetivos que pueden o no aplicarse a ciertos sustantivos. En la instrucción se les pide que subrayen los sustantivos de cada línea que se pueden emplear con los adjetivos de la primera columna. 


\begin{tabular}{|c|c|c|c|c|}
\hline ADJETIVOS & \multicolumn{4}{|c|}{ SUSTANTIVOS } \\
\hline Delicioso & reloj & café & comida & aroma \\
\hline Desabrido & bebida & ropa & corbata & habitación \\
\hline Dulce & vino & limón & música & voz \\
\hline Amargo & bebida & café & experiencia & auto \\
\hline Caliente & café & tarde & comida & habitación \\
\hline Cálido & música & abrazo & ambiente & risa \\
\hline Frío & saludo & sopa & día & helado \\
\hline Fresco & ropa & agua & música & concierto \\
\hline
\end{tabular}

Conforme a los niveles de evaluación basada en competencias podrían darse los siguientes casos o evidencias en el uso correcto de las anteriores colocaciones:

- Nivel receptivo: sabe la teoría pero no la sabe aplicar; es probablemente un alumno principiante. Es decir, escribe modelos pero no expresiones reales, por ejemplo: ropa fría / casa deliciosa.

- Nivel básico: resuelve bajo supervisión, con ayuda pero ya comienza a expresarse. Es decir, escribe modelos y expresiones reales con ayuda, por ejemplo: cerveza(s) fría(s), día(s) frío(s).

- Nivel Autónomo: resuelve solo pero únicamente en ciertos casos pues aunque ya sabe más todavía le pasan inadvertidos ciertos casos excepcionales o particulares. Es decir, escribe un mayor número de modelos y expresiones reales sin ayuda, por ejemplo: café delicioso, ropa fresca, comida caliente, etc.

- Nivel Estratégico: resuelve con todos los criterios y en todos los contextos porque es probablemente un alumno de nivel superior. Es decir, escribe modelos y expresiones sin ayuda alguna y teniendo en cuenta las condiciones de referencia y los criterios de calidad (comunicación correcta) en todo tipo de discurso, de tal suerte que puede decir, por ejemplo: día sombrío, carácter amargo, canción melancólica, etc.

Indudablemente, los alumnos en el aula muestran todos estos niveles de desempeño conforme avanzan en su aprendizaje. Más aún, si se considera el uso, la frecuencia y el sentido que pueden adquirir estas colocaciones en otros niveles de lengua como el coloquial, el pragmático y el cultural, se puede ver 
una correspondencia clara con los niveles de manejo de lengua determinados por el MCER: el nivel receptivo bien puede corresponder a los niveles de acceso (A1) y plataforma (A2), el nivel básico, al nivel umbral (B1), el nivel autónomo, a los niveles avanzado (B2) y de dominio (C1) y el nivel estratégico, al nivel de maestría (C2). Ello depende de la manera en que se trabaje con dichas colocaciones, la cantidad y tipo de ejemplos en las que se usen, y el enfoque con el cual se trabaje.

\section{CONCLUSIÓN}

La evaluación por competencias es el proceso mediante el cual se recopilan evidencias y se realiza un juicio o dictamen de las mismas con base en criterios preestablecidos que ayuden a determinar la actuación del aprendiente, le den retroalimentación y mejoren la idoneidad de la competencia adquirida.

La preparación de este tipo de evaluación no comienza por la determinación de las competencias mismas sino de los problemas que se plantean como contenido de los cursos o programas, para lo cual es necesario establecer una ruta que consiste primordialmente en:

a) desglosar las capacidades requeridas,

b) integrarlas en competencias que deben mejorar / evolucionar en el transcurso de un curso o programa, lo cual debe hacerse con base en

c) situaciones desafiantes y reales.

La consideración de la problemática determina las capacidades de respuesta que desarrollará el educando. El perfil de egreso supone considerar lo que puede ser más satisfactorio, útil y significativo para el futuro profesional, pues todo curso se desarrolla para lograr un perfil.

La evaluación deberá indicar el nivel de desempeño logrado (receptivo, básico, autónomo o estratégico) dado que un enfoque por competencias requiere de la enunciación de objetivos de desempeño y no solamente de actividades que refieren a la comprensión de la información o a su mera reflexión.

Entre las principales aportaciones de un enfoque por competencias se encuentra el volver a plantear el sentido y aplicación del aprendizaje en el contexto educativo. Obliga a determinar la finalidad de lo que se enseña y a decidir si únicamente se busca que los aprendientes retengan información y la reproduzcan en los esquemas y textos mostrados en la escuela o si, por el contrario, se pretende formar individuos con capacidades propias de 
razonamiento y poseedores de habilidades que les permitan resolver situaciones cotidianas de la vida real.

Sin duda, éste es el centro del debate de los paradigmas actuales en el campo de la didáctica, debate que permite vislumbrar lo difícil que es para un sistema educativo o de enseñanza -incluida la de lenguas vivas- y para su sistema de examinación, abandonar el planteamiento enciclopedista y depositario de la enseñanza y adoptar una visión utilitaria que permita resolver situaciones problemáticas de una manera eficaz y creativa.

En este sentido se requiere pasar de un modelo centrado en la información a un modelo centrado en desempeños. Se requiere "movilizar" la información, transferir las habilidades hacia las situaciones que surjan o puedan surgir. Un profesional no se desarrolla por la apropiación de la información que se le proporciona mediante las diferentes disciplinas desarticuladas entre sí, es necesario articularla en torno a objetos susceptibles de intervención: problemas específicos y tareas ejecutables definidos desde la realidad de la práctica de la profesión. 


\section{REFERENCIAS}

Delors, Jacques (1994). "Los cuatro pilares de la educación", en La Educación encierra un tesoro. México: El Correo de la UNESCO. Disponible en: https:// www.uv.mx/dgdaie/files/2012/11/CPP-DC-Delors-Los-cuatro-pilares.pdf

Díaz Barriga, Ángel. (2006). El enfoque de competencias en la educación. ¿Una alternativa o un disfraz de cambio? Perfiles Educativos, vol. XXVIII, núm. 111, enero-marzo, pp. 7-36. Instituto de Investigaciones sobre la Universidad y la Educación, Distrito Federal, México. Disponible en: http://www.redalyc.org/ articulo.oa?id=13211102

GARDNER, Howard. (1983). Multiple intelligences. Nueva York: Basic Books.

MARTENS (1997), citado por Sergio Tobón (2015). Formación Basada en Competencias, Pensamiento complejo, diseño curricular y didáctica. Recuperado el 27 de septiembre de 2018 de: https://www.uv.mx/psicologia/ files/2015/07/Tobon-S.-Formacion-basada-en-competencias.pdf

NúNez Chan, M.E. (2003). Guía para el diseño curricular por competencias. Documento de trabajo no publicado, Universidad Autónoma del Estado de México. Recuperado el 22 de octubre de 2018 de:

http://files.sld.cu/reveducmedica/files/2011/07/guia_para_el_diseno_ curricular_por_competencia.pdf

Organización para la Cooperación y el Desarrollo Económico (OCDE). (2002), Conocimientos y aptitudes para la vida. Resultados de PISA 2000, México, Santillana-OCDE.

Organización para la Cooperación y el Desarrollo Económico (OCDE). (2005). Informe PISA 2003. Aprender para el mundo de mañana. México, Santillana-OCDE

PerRenoud, Phillipe. (1999). Construir competencias desde la escuela. Santiago: Dolmen.

\section{Barcelona: Graó.}

Prosser, Gabriel.(2014).Competencias:Concepto, OrígenesyEvolución. Recuperado el 21 de septiembre de 2018 de: https://prezi.com/yuvad5×3mcqm/ competencias-concepto-origenes-y-evolucion/

QuesADA, D. (1998). Saber, opinión y ciencia: Una introducción a la teoría del conocimiento clásica y contemporánea. Barcelona: Ariel.

RoEgiers, Xavier. (2007). Pedagogía de la integración. Competencias e integración de los conocimientos en la enseñanza. Costa Rica. San José, Costa Rica: Coordinación Educativa y Cultural Centroamericana y AECl. Colección IDER. 
Universidad Autónoma de Barcelona. (2018). Herramientas de evaluación por competencias. Recuperado el 28 de septiembre de 2018 de: https://www. uab.cat/web/estudiar/mooc/planificar-y-disenar-un-mooc/herramientasde-evaluacion-1345668281394.html

ZabalA, Antoni. (2005). Las competencias en la educación escolar. Innovación educativa, Aula de Innovación Educativa. Núm. 161. Recuperado el 26 de septiembre de 2018 de: http://eoepsabi.educa.aragon.es/descargas/G_ Recursos_orientacion/g_7_competencias_basicas/g_7_1.docum. basicos/1.41.Ense\%F1ar_competencias.pdf 
